\title{
PERFORMANCE ANALYSIS OF THE MAP EQUALIZER WITH A PRIORI AND DISTRIBUTION OF THE EXTRINSIC LLRS
}

\author{
Noura Sellami ${ }^{1}$, Aline Roumy ${ }^{2}$, Inbar Fijalkow ${ }^{3}$ \\ ${ }^{1}$ ISECS, Route Menzel Chaker km 0.5, B.P 868, 3018 Sfax, Tunisia \\ ${ }^{2}$ IRISA-INRIA, Campus de Beaulieu, 35042 Rennes Cedex, France \\ ${ }^{3}$ Laboratoire ETIS, UMR 8051, ENSEA-UCP-CNRS, \\ 6 av. du Ponceau, 95014 Cergy-Pontoise, France
}

\begin{abstract}
To combat the effects of intersymbol interference, the optimal equalizer to be used is based on maximum a posteriori (MAP) detection. We consider the case where the MAP equalizer is provided with a priori information on the transmitted data, as in a turbo equalizer. We propose to study analytically the impact of the a priori on the MAP equalizer performance. We distinguish two cases: the case of unreliable $a$ priori and the case of reliable a priori. We show that when the a priori is reliable, it is better to perform the detection of the transmitted symbols based on the a posteriori outputs of the MAP equalizer rather than on the a priori only. In a turbo equalizer, the equalizer and the decoder exchange extrinsic Log Likelihood Ratios (LLRs). We propose here to study analytically their distribution. The aim of this study is to perform in the future the analytical convergence analysis of turbo equalizers using the MAP equalization.
\end{abstract}

\section{INTRODUCTION}

To combat the effects of intersymbol interference (ISI), an equalizer has to be used. The optimal equalizer, in the sense of minimum sequence error rate (SER) or bit error rate (BER) is based on maximum a posteriori (MAP) detection. We distinguish two criteria, the MAP sequence detection and the MAP symbol detection. When no a priori information on the transmitted data is available, MAP detection turns into maximum likelihood (ML) detection. Efficient algorithms exist for MAP sequence detection, for example the SER optimizing Viterbi algorithm [1], and MAP symbol detection, for example the BER optimizing BCJR algorithm [2]. These algorithms are interesting since their complexity grows linearly rather than exponentially with the sequence size.

The performance of the Viterbi equalizer in the presence of additive white Gaussian noise (AWGN) has been studied by Forney [1]. This study assumed that the channel is perfectly known at the receiver and no a priori information is provided to the equalizer.

In this paper, we consider the case where the equalizer has a priori information on the data, provided by another module in the receiver, for instance the decoder in a turbo-equalizer [3]. In [4], we analyzed the impact of the a priori information on the MAP equalizer performance. Since the results presented in [4] hold only for unreliable a priori, we propose here to extend the analysis to the case of reliable a priori. The study in [4] has been performed considering the a posteriori probabilities at the output of the equalizer. However, the basic idea behind iterative processing is to exchange extrinsic probabilities among the equalizer and the decoder rather than a posteriori probabilities, in order to achieve successively refined performance [3]. Therefore, we propose in this paper to extend the study of [4] to the extrinsic outputs. Thus, we derive the expression of the distribution of the extrinsic Log Likelihood Ratios (LLRs) at the output of the equalizer when it is provided with Gaussian a priori observations, in both cases of reliable and unreliable $a$ priori. The aim of this study is to perform, in a future work, the convergence analysis of a turbo equalizer in an analytical way. Actually, most analyses are based on extrinsic information transfer (EXIT) charts [5][6]. These analyses use generally simulations since it is difficult to study analytically the performance of a MAP equalizer having a large number of states.

The paper is organized as follows. In section 2, we describe the system model. In section 3 , we study the impact of the a priori information on the equalizer performance and give the distribution of the extrinsic LLRs at its output. In section 4 , we give simulation results.

Throughout this paper scalars and matrices are lower and upper case respectively and vectors are underlined lower case. $(.)^{T}$ denotes the transposition.

\section{SYSTEM MODEL}

We consider a data transmission system over a frequency selective channel. The input information bit sequence is mapped to the symbol alphabet $\mathcal{A}$. For simplicity, we will consider only the BPSK modulation $(\mathcal{A}=\{+1,-1\})$. We assume that transmissions are organized into bursts of $T$ symbols. The channel is supposed to be invariant during one burst. The received baseband signal sampled at the symbol rate at time $k$ is

$$
x_{k}=\sum_{l=0}^{L-1} h_{l} s_{k-l}+n_{k}
$$

where $L$ is the channel memory and $s_{k}$, for $1-L \leq k \leq$ $T-1$, are the transmitted symbols. In this expression, $n_{k}$ are modeled as independent samples of a real white Gaussian 
noise with normal probability density function (pdf) $\mathcal{N}\left(0, \sigma^{2}\right)$ where $\mathcal{N}\left(\alpha, \sigma^{2}\right)$ denotes a Gaussian distribution with mean $\alpha$ and variance $\sigma^{2}$. The term $h_{l}$ is the $l^{\text {th }}$ tap gain of the channel, which is assumed to be real valued. Let $\underline{s}=\left(s_{T-1}, \ldots, s_{1-L}\right)^{T}$ be the $(L+T-1)$-long vector of symbols and $\underline{n}=\left(n_{T-1}, \ldots, n_{0}\right)^{T}$ be the $T$-long noise vector. The output of the channel is the $T$-long vector $\underline{x}=\left(x_{T-1}, \ldots, x_{0}\right)^{T}$ defined as

$$
\underline{x}=\tau(h) \underline{s}+\underline{n}
$$

where $\tau(h)$ is a $T \times(T+L-1)$ Toeplitz matrix with its first row being $\left(h_{0}, h_{1}, \ldots, h_{L-1}, 0, \ldots, 0\right)$ and its first column $\left(h_{0}, 0, \ldots, 0\right)^{T}$.

When the channel is known and no a priori information is provided to the equalizer, the data estimate according to the MAP sequence criterion (or to the ML criterion since there is no $a$ priori) is given by

$$
\underline{\hat{s}}=\arg \min _{\underline{u}}\left(\|\underline{x}-\tau(h) \underline{u}\|: \underline{u} \in \mathcal{A}^{T+L-1}\right) .
$$

Now, we consider a particular error event characterized by its length $m$ [1]. Thus, we suppose that there exists an interval of size $m$ such that all the symbols of $\underline{\hat{s}}$ are different from the corresponding symbols of $\underline{s}$ while the preceding symbol and the following one are the same for $\underline{s}$ and $\underline{\hat{s}}$. Define $\underline{s}_{m}$ and $\underline{\hat{s}}_{m}$ to be the vectors of symbols corresponding to this interval and the vector of errors $\underline{e}_{m}=\underline{\hat{s}}_{m}-\underline{s}_{m}$. A subevent $\mathcal{E}_{m}$ of the error event is that $\underline{\hat{s}}_{m}$ is better than $\underline{s}_{m}$ in the sense of the ML metric

$$
\mathcal{E}_{m}:\left\|\underline{x}_{m}-\tau_{m}(h) \underline{\hat{s}}_{m}\right\| \leq\left\|\underline{x}_{m}-\tau_{m}(h) \underline{s}_{m}\right\|
$$

where $\underline{x}_{m}$ is the subvector of $\underline{x}$ and $\tau_{m}(h)$ is the block of $\tau(h)$ corresponding to the error interval. The probability $P\left(\mathcal{E}_{m}\right)$ of $\mathcal{E}_{m}$ is given by [1]:

$$
P\left(\mathcal{E}_{m}\right)=Q\left(\frac{\left\|\underline{\varepsilon}_{m}\right\|}{2 \sigma}\right)
$$

where $\underline{\varepsilon}_{m}=\tau_{m}(h) \underline{e}_{m}$ and $Q(\alpha)=\frac{1}{\sqrt{2 \pi}} \int_{\alpha}^{\infty} \exp \left(-y^{2} / 2\right) d y$. Let $\Sigma_{m}$ be the set of all possible error events of length $m$. Then, the probability, $P\left(\Sigma_{m}\right)$, that any error event is of length $m$ is bounded by the sum of the probabilities of the subevents $\mathcal{E}_{m}$

$$
P\left(\Sigma_{m}\right) \leq \sum_{\mathcal{E}_{m}} P\left(\mathcal{E}_{m}\right) .
$$

Let $d_{\min }$ be the channel minimum distance [1]. Because of the exponential decrease of the complementary Gaussian distribution function $Q($.$) , the overall probability of error P(\Sigma) \leq$ $\sum_{m} P\left(\Sigma_{m}\right)$ will be dominated at high SNR by the term involving the minimum value $d_{\min }$ of $\left\|\underline{\varepsilon}_{m}\right\|$. Thus,

$$
P(\Sigma) \simeq Q\left(\frac{d_{\min }}{2 \sigma}\right) .
$$

\section{ANALYSIS OF THE EQUALIZER}

We assume here that the channel is perfectly known at the receiver. The MAP equalizer computes the a posteriori probabilities (APPs) on the transmitted bits, $P\left(s_{k}=s \mid \underline{x}\right), s \in \mathcal{A}$,
$1-L \leq k \leq T-1$. As in a turbo equalizer, we assume that it outputs the extrinsic log-likelihood ratios (LLRs) [6]:

$$
\begin{aligned}
\operatorname{LLR}_{\text {ext_eq }}\left(s_{k}\right) & =L L R_{e q}\left(s_{k}\right)-L L R_{\text {priori_eq }}\left(s_{k}\right) \\
& =\log \frac{P\left(s_{k}=+1 \mid \underline{x}\right)}{P\left(s_{k}=-1 \mid \underline{x}\right)}-\log \frac{P\left(s_{k}=+1\right)}{P\left(s_{k}=-1\right)}
\end{aligned}
$$

which are the a posteriori LLRs $L L R_{e q}\left(s_{k}\right)$ minus the a priori $L L R_{\text {priori_eq }}\left(s_{k}\right)$. We suppose that the a priori observations at the input of the equalizer are modeled as the outputs of an AWGN (Additive White Gaussian Noise) channel with zero mean and variance $\sigma_{e q}^{2}$. Hence, the a priori LLRs are modeled as i.i.d samples from a random variable with the conditional pdf $\mathcal{N}\left(\frac{2 s_{k} \mu_{e q}^{2}}{\sigma^{2}}, \frac{4 \mu_{e q}^{2}}{\sigma^{2}}\right)$ where $\mu_{e q}=\frac{\sigma}{\sigma_{e q}}$. This assumption is classically taken in the analysis of iterative receivers [5][6]. We showed in [4] that when the equalizer is provided with the a priori information, the probability of the error event $P\left(\mathcal{E}_{m}\right)$ is given by

$$
P\left(\mathcal{E}_{m}\right)=Q\left(\frac{\sqrt{\left\|\underline{\varepsilon}_{m}\right\|^{2}+4 m \mu_{e q}^{2}}}{2 \sigma}\right) .
$$

In the following, we propose to calculate the overall probability of error of the equalizer and to find the distribution of the extrinsic LLRs $L L R_{e x t_{-} e q}\left(s_{k}\right)$. We generalize the result of [4] and distinguish two cases: the case of unreliable a priori information ( $\mu_{e q}$ is low) and the case of reliable a priori $\left(\mu_{e q}\right.$ is high).

\subsection{Case1: unreliable a priori}

We consider here the case of unreliable a priori. By definition, $\left\|\underline{\varepsilon}_{m}\right\|^{2} \geq d_{\min }^{2}$. Generally, in the MAP equalizer, errors occur in packets. Thus, we do not consider isolated errors since they occur rarely and we assume that $m \geq 2$. Then, an upper bound for $P\left(\mathcal{E}_{m}\right)=Q\left(\frac{\sqrt{\left\|\underline{\varepsilon}_{m}\right\|^{2}+4 m \mu_{e q}^{2}}}{2 \sigma}\right)$ is obtained by replacing $\left\|\underline{\varepsilon}_{m}\right\|^{2}$ by $d_{\min }^{2}$ and $m$ by 2 . We observed that this bound is reached for channels with memory $L$ less than 6, since for these channels the error sequence allowing to attain the minimum distance is of length $m=2$ (see examples of channels in [7]). For longer channels, an exhaustive search of a close upper bound has to be performed. For the simplicity of the presentation, we will consider in the following the case of short channels $(L \leq 6)$ since we have the exact expression of the bound in this case. At high SNR, the term corresponding to the upper bound will dominate the sum of the probabilities of the error events (because of the exponential decrease of the $Q$ (.) function). Thus the overall probability of error can be approximated by [4]:

$$
P(\Sigma) \simeq Q\left(\frac{\sqrt{d_{\min }^{2}+8 \mu_{e q}^{2}}}{2 \sigma}\right) .
$$

We know that the overall probability of error for the BPSK modulation, for an AWGN channel with zero mean and variance $\sigma_{1}^{2}$ is 


$$
P(\Sigma) \simeq Q\left(\frac{1}{\sigma_{1}}\right) .
$$

Comparing (9) and (10), we can conclude that the performance of the MAP equalizer when it is provided with the $a$ priori information is equivalent to the performance achieved for an AWGN channel with zero mean noise and variance $\sigma_{1}^{2}=\frac{4 \sigma^{2}}{d_{\min }^{2}+8 \mu_{e q}^{2}}$.

Hence, the a posteriori LLRs at the output of the MAP equalizer can be modeled as i.i.d samples from a random variable with pdf $\mathcal{N}\left(\frac{2 s_{k}}{\sigma_{1}^{2}}, \frac{4}{\sigma_{1}^{2}}\right)$

Since the a priori and extrinsic LLRs are independent by construction, we obtain

$\operatorname{LLR}_{\text {ext_eq }}\left(s_{k}\right) \sim \mathcal{N}\left(s_{k}\left(\frac{2}{\sigma_{1}^{2}}-\frac{2 \mu_{e q}^{2}}{\sigma^{2}}\right),\left(\frac{4}{\sigma_{1}^{2}}-\frac{4 \mu_{e q}^{2}}{\sigma^{2}}\right)\right)$

Hence,

$L L R_{e x t_{-} e q}\left(s_{k}\right) \sim \mathcal{N}\left(s_{k}\left(\frac{d_{\min }^{2}+4 \mu_{e q}^{2}}{2 \sigma^{2}}\right),\left(\frac{d_{\min }^{2}+4 \mu_{e q}^{2}}{\sigma^{2}}\right)\right)$

\subsection{Case2: reliable $a$ priori}

When the a priori become good ( $\mu_{e q}$ is high), the a priori observations have more influence on the detection than the channel observations. Since the a priori information are independent, the errors will not occur in packets. Thus, in this case, isolated errors $\left(m=1\right.$ and $\left.\left\|\underline{\varepsilon}_{m}\right\|^{2}=4\right)$ will occur and will dominate the overall probability of error which can be approximated by:

$$
P(\Sigma) \simeq Q\left(\frac{\sqrt{1+\mu_{e q}^{2}}}{\sigma}\right) .
$$

In this case, the performance of the MAP equalizer is equivalent to the performance of an AWGN channel with zero mean and variance $\sigma_{2}^{2}=\frac{\sigma^{2}}{\left(1+\mu_{e q}^{2}\right)}$. Note that the performance is independent from the channel.

Assuming that the a priori and extrinsic LLRs are independent, we obtain

$\operatorname{LLR}_{\text {ext } t_{-} q}\left(s_{k}\right) \sim \mathcal{N}\left(s_{k}\left(\frac{2}{\sigma_{2}^{2}}-\frac{2 \mu_{e q}^{2}}{\sigma^{2}}\right),\left(\frac{4}{\sigma_{2}^{2}}-\frac{4 \mu_{e q}^{2}}{\sigma^{2}}\right)\right)$

Hence,

$$
\operatorname{LLR}_{\text {ext_eq }}\left(s_{k}\right) \sim \mathcal{N}\left(s_{k}\left(\frac{2}{\sigma^{2}}\right), \frac{4}{\sigma^{2}}\right)
$$

We notice that when the a priori information become good, the extrinsic LLRs are equivalent to the LLRs corresponding to the AWGN channel with zero mean and variance $\sigma^{2}$. Hence, the effect of the ISI is eliminated. In a turbo equalizer, the equalizer provides the decoder with the extrinsic LLRs
$L L R_{\text {ext_eq }}\left(s_{k}\right)$, at each iteration. Hence, when the a priori information are reliable, the performance of the decoder (which is also the performance of the turbo equalizer) is equivalent to the performance of the coded AWGN channel.

\subsection{Extrinsic LLRs modeling}

Until now, we give two distributions of the extrinsic LLRs in two limit cases, i.e. when the a priori is not reliable ( $\mu_{e q}$ is low) and when it is reliable ( $\mu_{e q}$ is high). However, it is not obvious that the analysis holds for the intermediate values of $\mu_{e q}$. We suggest here that there exists a limit value $\mu_{e q-\lim }$ of $\mu_{e q}$ such as

$$
\left\{\begin{array}{l}
L L R_{\text {ext_eq }}\left(s_{k}\right) \sim \mathcal{N}\left(s_{k}\left(\frac{d_{\min }^{2}+4 \mu_{e q}^{2}}{2 \sigma^{2}}\right),\left(\frac{d_{\min }^{2}+4 \mu_{e q}^{2}}{\sigma^{2}}\right)\right) \\
, \text { if } \mu_{e q}<\mu_{e q-\lim } \\
L L R_{\text {ext_eq }}\left(s_{k}\right) \sim \mathcal{N}\left(s_{k}\left(\frac{2}{\sigma^{2}}\right), \frac{4}{\sigma^{2}}\right), \text { elsewhere. }
\end{array}\right.
$$

This value corresponds to the equality between expressions (11) and (12). Thus, it is given by

$$
\mu_{e q_{-} \lim }=\sqrt{1-\frac{d_{\min }^{2}}{4}}
$$

This result will be validated by the simulations in the next section.

\subsection{Usefulness of the MAP equalizer when the $a$ priori is reliable}

When the a priori is reliable, a question that one can ask is: is it better to perform the detection of the transmitted symbols based on the a posteriori outputs of the MAP equalizer or based on the a priori only? i.e. does the equalizer benefit from the channel observations? In order to answer this question, we propose to compare the probabilities of error in both cases.

We first consider the case where the detection is based on the a priori only. Since the a priori observations at the input of the equalizer are modeled as the outputs of an AWGN with variance $\sigma_{e q}^{2}$, the overall probability of error is

$$
P(\Sigma) \simeq Q\left(\frac{1}{\sigma_{e q}}\right) .
$$

When the MAP equalizer is used, the overall probability of error is (see section 3.2)

$$
\begin{aligned}
P(\Sigma) & \simeq Q\left(\frac{\sqrt{1+\mu_{e q}^{2}}}{\sigma}\right) \\
& =Q\left(\sqrt{\frac{1}{\sigma^{2}}+\frac{1}{\sigma_{e q}^{2}}}\right) .
\end{aligned}
$$

Since $Q($.$) is monotonically decreasing, the probability given$ in (14) is greater than the one given in (15). Thus, in the case of reliable a priori, in terms of error probability, it is better to use the MAP equalizer rather than performing the detection using only the a priori. 


\section{SIMULATION RESULTS}

In this section, we propose to test for the validity of the analytical results given previously. In the simulations, the modulation used is the BPSK and the channel is assumed to be constant and known at the receiver. We consider the following channels [7]:

- Channel3: $(0.5 ; 0.71 ; 0.5)$

- Channel5: $(0.29 ; 0.50 ; 0.58 ; 0.50 ; 0.29)$.

The minimum distances of Channel 3 and Channel5 are respectively 1.5308 and 1.0532 [7]. We provide the equalizer with Gaussian a priori LLRs with the conditional pdf $\mathcal{N}\left(\frac{2 s_{k} \mu_{e q}^{2}}{\sigma^{2}}, \frac{4 \mu_{e q}^{2}}{\sigma^{2}}\right)$, for a given $\mu_{e q}=\frac{\sigma}{\sigma_{e q}}$. We perform the detection of the transmitted symbols using the extrinsic LLRs at the output of the equalizer. In Figures 1 and 2, we plot the Bit Error Rate $(B E R)$ curves with respect to the SNR, for different values of the ratio $\mu_{e q}$, respectively for Channel3 and Channel5. Each curve is obtained while the ratio $\mu_{e q}$ is kept constant. The solid lines indicate the performance obtained by simulations. The dotted lines are obtained by considering the theoretical expressions of the extrinsic LLRs given in (11) and (12). By using (13), we obtain that for Channel 3, $\mu_{e q-\lim }=0.64$ and for Channel 5, $\mu_{e q-\lim }=0.866$.

We notice that, for both channels, when $\mu_{e q} \geq \mu_{e q-\lim }$, the performance is similar to the performance of an AWGN channel with variance $\sigma^{2}$. We notice that when $\mu_{e q}<\mu_{e q-\lim }$, the theoretical curves approximate well the curves obtained by simulations for high SNR. For low SNR, the approximation is less accurate. Actually, for $\mu_{e q}<\mu_{e q-\lim }$, the assumption that the LLRs at the output of the MAP equalizer have pdf $\mathcal{N}\left(\frac{2 s_{k}}{\sigma_{e q}^{2}}, \frac{4}{\sigma_{e q}^{2}}\right)$ is not always verified.

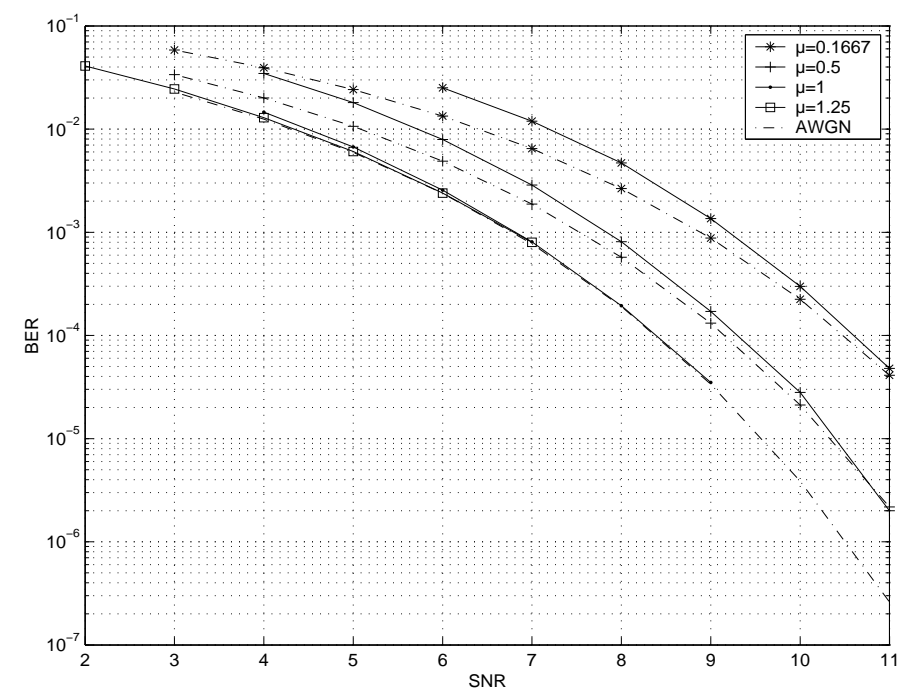

Fig. 1. BER versus SNR: comparison of the equalizer performance (solid curves) and the theoretical performance (dotted curves) for Channel3 $\left(\mu_{e q-\lim }=0.64\right)$, when the detection is based on the extrinsic LLRs.

Figures 3 and 4 show the means of the extrinsic LLRs when the transmitted bits are equal to +1 with respect to $\mu_{e q}$

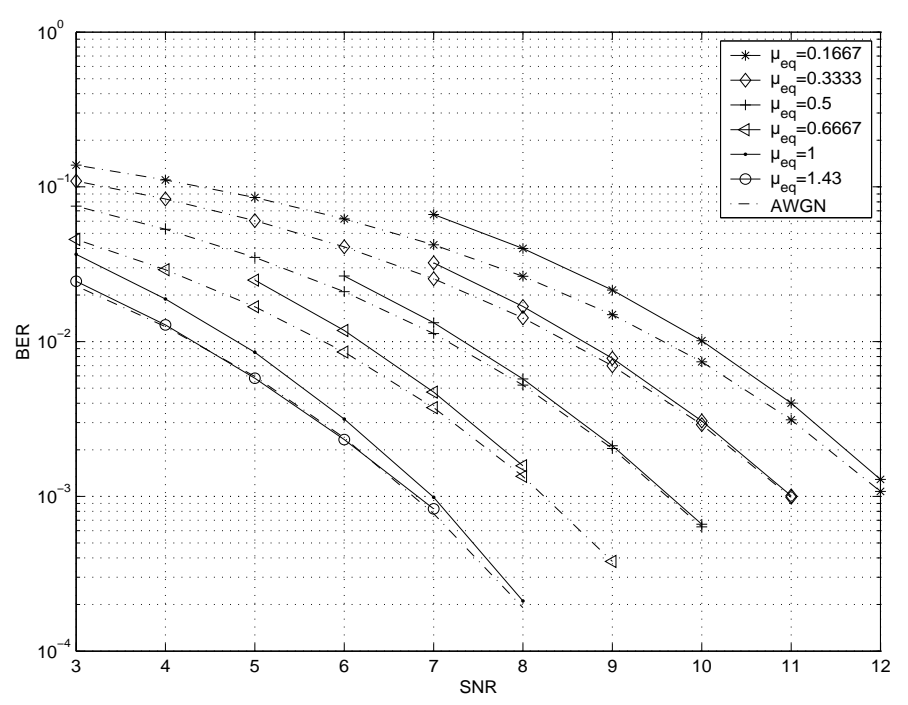

Fig. 2. BER versus SNR: comparison of the equalizer performance (solid curves) and the theoretical performance (dotted curves) for Channel5 $\left(\mu_{e q-\lim }=0.866\right)$, when the detection is based on the extrinsic LLRs.

respectively for Channel 3 at $\mathrm{SNR}=9 \mathrm{~dB}$ and for Channel 5 at $\mathrm{SNR}=7 \mathrm{~dB}$. We notice that for $\mu_{e q}>>\mu_{e q-\lim }$ and $\mu_{e q}<<$ $\mu_{e q-\lim }$, the analytical means approximate well the means obtained by simulations. Around the limit value $\mu_{e q-\lim }$ the approximation is less accurate, but is still satisfactory to predict the extrinsic performance of the equalizer as shown in Figures 1 and 2.

\section{CONCLUSION}

In this paper, we consider a MAP equalizer provided with Gaussian a priori observations, as in a turbo equalizer. We propose to study analytically the impact of the a priori information on the MAP equalizer performance. We distinguish two cases: the case of unreliable a priori and the case of reliable a priori. We show that when the a priori is reliable, it is better in terms of performance to carry out the detection of the transmitted symbols based on the a posteriori outputs of the MAP equalizer rather than on the a priori only. We also give an expression of the distribution of the extrinsic LLRs at the output of the MAP equalizer. The aim of this work is to perform in the future the analytical convergence analysis of turbo equalizers using MAP equalization. This requires us to perform also the study of the extrinsic LLRs at the output of the decoder.

\section{REFERENCES}

[1] G.D.Forney, Jr., "Maximum-likelihood sequence estimation for digital sequences in the presence of intersymbol interference," IEEE Trans. Inf. Theory, vol. 18, pp. 363378 , May 1972.

[2] L.R.Bahl, J.Cocke, F.Jelinek, and J.Raviv, "Optimal decoding of linear codes for minimizing symbol error rate," 
IEEE Trans. Inf. Theory, vol. IT-32, pp.284-287, March 1974.

[3] C.Douillard, M.Jézéquel, C.Berrou, A.Picart, P.Didier, and A.Glavieux, "Iterative correction of intersymbol interference: turbo-equalization," European Trans. Telecommun., vol. 6, no. 5, pp. 507-511, 1995.

[4] N.Sellami, A.Roumy, and I.Fijalkow, "On the analysis of the MAP equalizer performance within an iterative receiver, "Signal Process. Advances in Wireless Comm. (SPAWC'04), Lisbon, Portugal, July 2004.

[5] S.Ten Brink, "Convergence of iterative decoding," IEEE Electronic Letters, vol. 35, pp.806-808, May 1999.

[6] M.Tüchler, R.Koetter, and A.Singer, "Turbo equalization: principles and new results," IEEE Trans. on Comm., vol. 50, no. 5, pp. 754-767, May 2002.

[7] W.Ser, K.Tan, and K.Ho, "A new method for determining "unknown" worst-case channels for maximum-likelihood sequence estimation," IEEE Trans. on Comm., vol. 46, no. 2, pp. 164-168, February 1998.

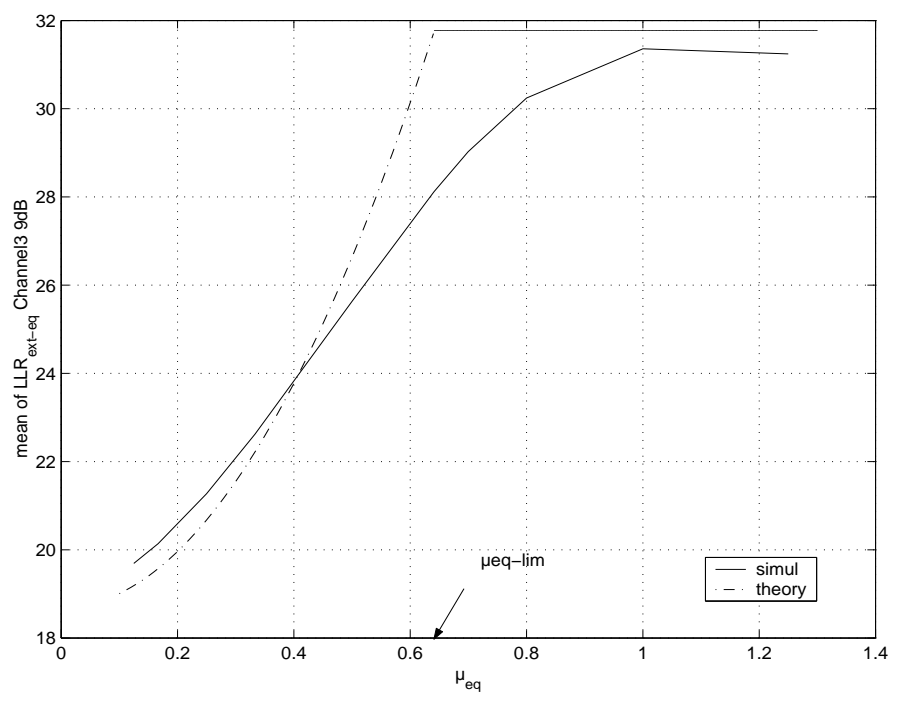

Fig. 3. Means of the extrinsic LLRs when the transmitted bits are equal to +1 with respect to $\mu_{e q}$ for Channel3 and $\mathrm{SNR}=9 \mathrm{~dB}$.

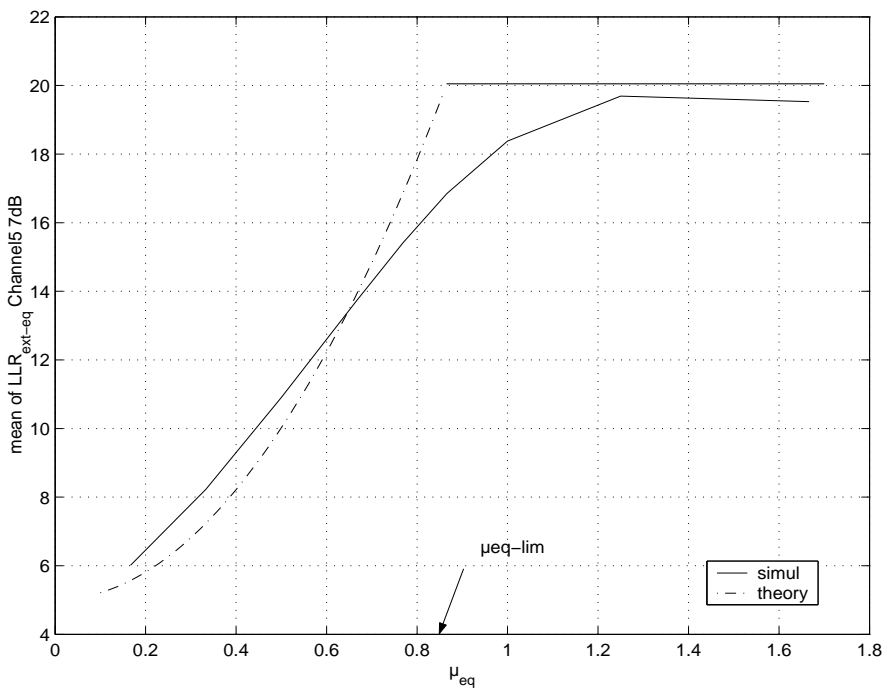

Fig. 4. Means of the extrinsic LLRs when the transmitted bits are equal to +1 with respect to $\mu_{e q}$ for Channel5 and $\mathrm{SNR}=7 \mathrm{~dB}$. 